\title{
Peripapillary Neovascular Membrane in a Young Pregnant Woman and Prompt Response to Ranibizumab Injections following Uneventful Delivery
}

\author{
Konstantinos Anastasilakis Chrysanthos Symeonidis \\ Konstantinos Kaprinis Asimina Mataftsi \\ Argyrios Tzamalis Stavros A. Dimitrakos \\ 2nd Department of Ophthalmology, Aristotle University of Thessaloniki, \\ Thessaloniki, Macedonia, Greece
}

\section{Key Words}

Choroidal neovascularization · Pregnancy · Ranibizumab

\begin{abstract}
Purpose: Occurrence of choroidal neovascularization (CNV) during pregnancy has been reported as a complication of presumed ocular histoplasmosis syndrome or punctuate inner chorioretinopathy. To our knowledge, idiopathic CNV (ICNV) during pregnancy has only been reported once in the relevant literature. Bevacizumab has been used for the treatment of ICNV in small case series. However, there is limited experience regarding the use of ranibizumab for the management of ICNV.

Case Report: A 31-year-old woman in the eighth month of her second pregnancy was diagnosed with mild macular and papillary edema. She was followed up using biomicroscopy, fluorescein angiography (FA), and optical coherence tomography (OCT). After 3 months, visual acuity further deteriorated and funduscopy, FA and OCT findings revealed a juxtapapillary choroidal neovascular membrane (CNVM). After two ranibizumab injections, best-corrected visual acuity increased significantly, physiological macular anatomy was restored and no subretinal fluid was observed.

Discussion: In this case report, we present a young pregnant patient with peripapillary ICNV and neurosensory detachment involving the macula, and treatment of the eye with intravitreal ranibizumab following uneventful delivery. Increased angiogenic factor levels associated with pregnancy may contribute to the onset of CNV although this relationship has to be investigated experimentally. The rapid response to ranibizumab suggests that this anti-VEGF agent may be an alternative treatment option in the management of peripapillary ICNV.
\end{abstract}




\section{Introduction}

Several ocular conditions have been reported to be influenced by pregnancy. This is attributed to hormonal alterations, changing hemodynamic parameters and increased expression of angiogenic growth factors associated with gestation [1]. These events may affect retinal pigment epithelium and the physiology of the choriocapillaris and potentially increase the risk of choroidal neovascularization $(\mathrm{CNV})$ development.

$\mathrm{CNV}$ occurrence during pregnancy has been reported as a complication of presumed ocular histoplasmosis syndrome [1] or punctuate inner chorioretinopathy [2]. To our knowledge, idiopathic CNV (ICNV) during pregnancy has only been reported once in the relevant literature [1]. Bevacizumab has been used for the treatment of ICNV in small case series $[3,4]$. However, there is limited experience regarding ranibizumab use for the management of ICNV. We report a case of peripapillary ICNV and its treatment with intravitreal ranibizumab in a healthy, young, pregnant woman.

\section{Case Report}

A 31-year-old woman in the eighth month of her second pregnancy was referred to our clinic for blurred vision OD. Ophthalmic history was unremarkable. She reported one previous uncomplicated pregnancy. Best-corrected visual acuity (BCVA) was 20/25 OD, 20/20 OS, with no metamorphopsia OU. Fundoscopy OD revealed mild macular and papillary edema and a slightly elevated optic disc OS. The patient had neither proteinuria nor hypertension, and pre-eclampsia was excluded. Because of her pregnancy, fluorescein angiography (FA) was not performed. As BCVA was minimally decreased, no intervention and frequent follow-ups were recommended. After uneventful labor, an infant with no significant health problems was delivered. Two months later, lactation was stopped.

Three months after her last ophthalmic examination, BCVA was 20/32 with metamorphopsia OD and 20/20 OS. Fundoscopy revealed a serous neurosensory detachment in the papillomacular region that involved the fovea and subretinal hemorrhage inferotemporally of the disc OD, but no significant findings OS. FA and optical coherence tomography (OCT) scans showed a juxtapapillary choroidal neovascular membrane (CNVM) OD, but observation was again considered sufficient, given the acceptable BCVA level. A month later BCVA was 20/50 OD and subretinal fluid (SRF) was increased. This observation was confirmed by OCT and indocyanine green (ICG) angiography (fig. 1). Intravitreal ranibizumab treatment was discussed with and accepted by the patient. BCVA improved 1 week (20/32) and 1 month after the first injection (20/25). FA showed decreased CNVM size and peripapillary leakage. OCT confirmed restoration of physiological macular anatomy with a small, shallow peripapillary neurosensory detachment. Due to persistent SRF, a second ranibizumab injection was performed. One month after the second injection, BCVA was 20/20 with slight metamorphopsia. A small quantity of residual peripapillary SRF and initiation of scarring in the papillomacular region were observed. Two months after the second injection, BCVA was 20/20 with slight metamorphopsia and no SRF (fig. 2).

\section{Discussion}

Vascular endothelial growth factor (VEGF) is regarded as the primary regulator of angiogenesis. After conception, VEGF and placental growth factor (PlGF) are highly expressed in the placenta, and can be detected in the maternal circulation throughout pregnancy [5]; findings which are possibly indicative of a role in the pathogenesis of pregnancy-associated ocular CNVM as presented in this case. 
The Macular Photocoagulation Study Group proposed argon laser photocoagulation in patients with extrafoveal ICNV, excluding those located in the papillomacular bundle [6]. ICNV is not necessarily associated with marked visual deterioration and its prognosis in young patients that have not received any treatment is often good [7], favoring a conservative treatment strategy. In our case, intravitreal ranibizumab administration was based on previous reports of anti-VEGF effectiveness regarding BCVA improvement and macular edema reduction in elderly patients with idiopathic CNV $[3,4]$. As there are no anti-VEGF treatment guidelines for peripapillary ICNV in young patients, continuation of treatment was decided based on rapid BCVA deterioration as well as funduscopic, FA and OCT findings $[3,4]$.

In this case report, we present a young pregnant patient with peripapillary ICNV and neurosensory detachment involving the macula. It is possible that increased angiogenic factor levels associated with pregnancy may contribute to the onset of CNV, although this relationship has to be investigated experimentally. The safety and efficacy of ranibizumab in this context is yet to be determined in a large series of patients. However, the rapid response to this anti-VEGF agent in our case (within 1 week from the first injection) suggests that it may be an alternative treatment option in the management of pregnancyassociated peripapillary ICNV.

\section{Disclosure Statement}

The authors report no proprietary interest or financial support. 

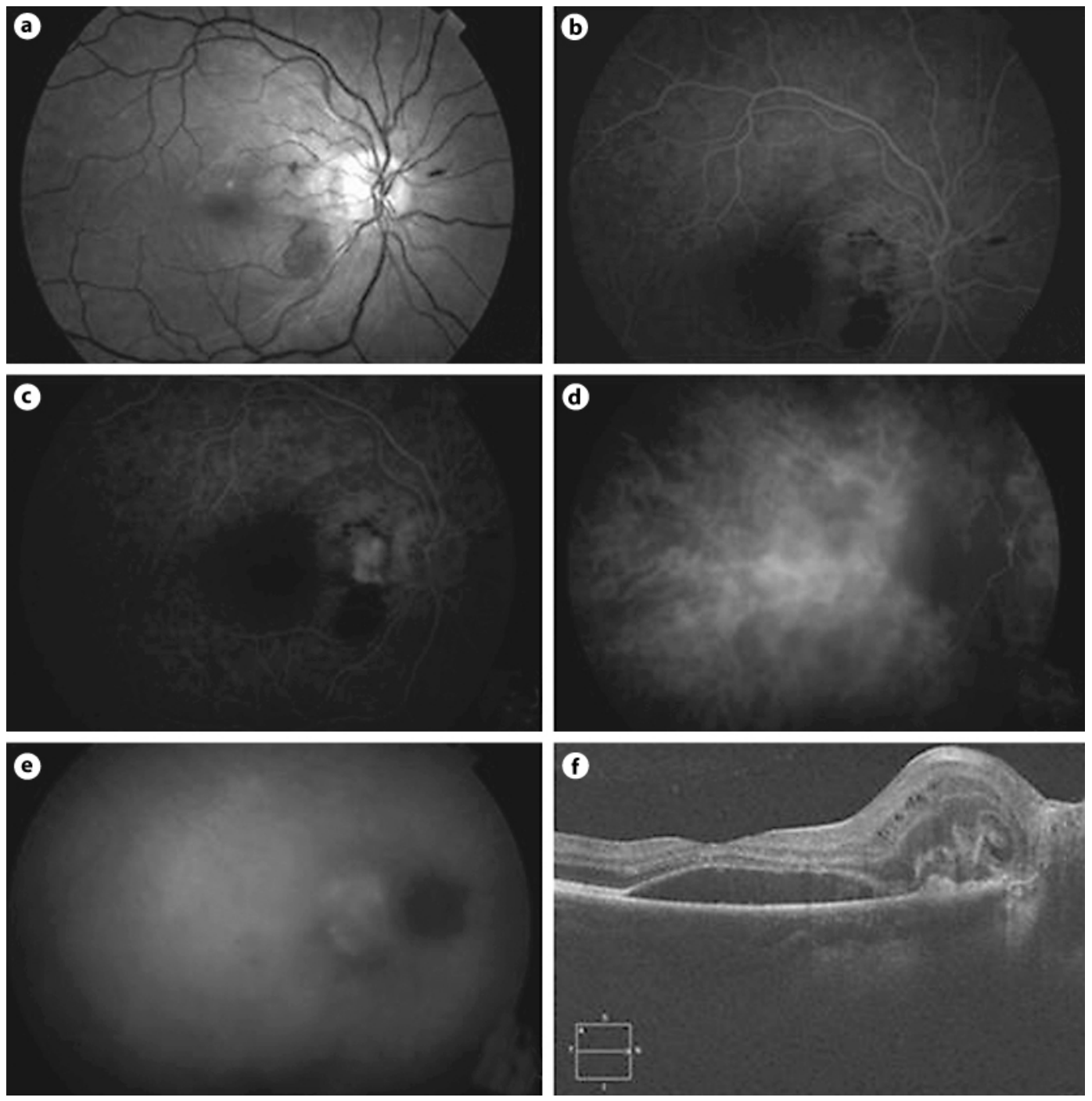

Fig. 1. FA, ICG angiography and OCT of the right eye after uneventful delivery. a Red-free image. Serous neurosensory detachment in the papillomacular region, involving the fovea and a localized area of subretinal hemorrhage inferotemporally of the optic disc. b FA, early phase. A hyperfluorescent, welldefined juxtapapillary CNVM is shown. c FA, late phase. Fluorescein pooling and staining of the neovascular complex can be observed. d ICG, early phase. A hypofluorescent lesion is depicted. e ICG, late phase. Staining of the neovascular complex with leakage in the subretinal space. $f$ OCT revealing a neurosensory detachment with an adjacent area of high reflectance indicating juxtapapillary type II CNV. 

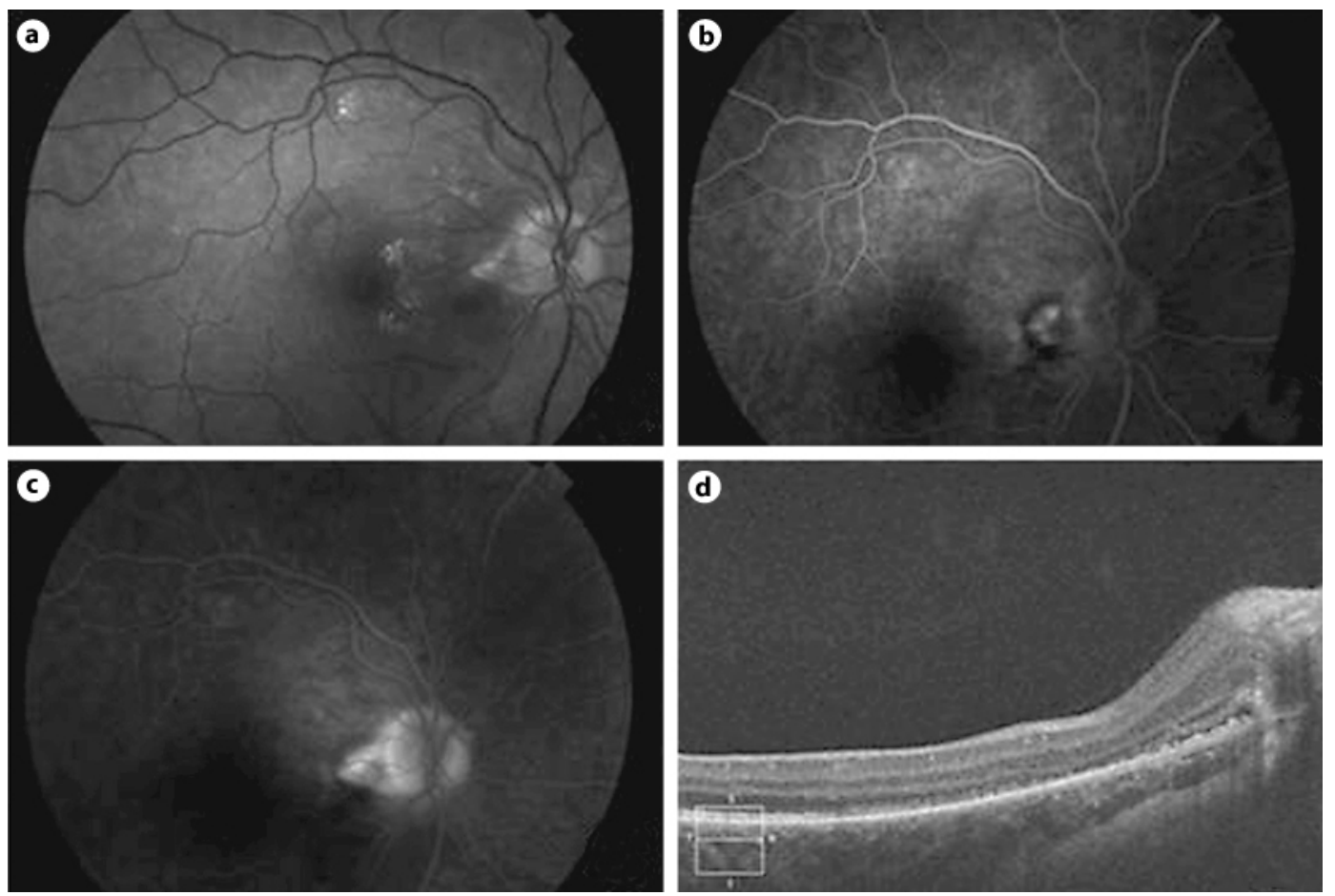

Fig. 2. FA and OCT of the right eye 1 month after the first intravitreal ranibizumab injection. a Redfree image. Resolution of the neurosensory detachment. A ring of hard exudates surrounds the temporal rim of the disc. b FA. Reduction in size of the neovascular complex. c FA, late phase. Decreased peripapillary leakage. $\mathbf{d}$ OCT confirming restoration of the physiological macular anatomy with a small remaining area of reflectance next to the optic disc and an adjacent shallow neurosensory detachment surrounding the lesion.

\section{References}

1 Rhee P, Dev S, Mieler WF: The development of choroidal neovascularization in pregnancy. Retina 1999;19:520-524.

2 Sim DA, Sheth HG, Kaines A, Tufail A: Punctate inner chorioretinopathy-associated choroidal neovascular membranes during pregnancy. Eye (Lond) 2008;22:725-727.

3 Mandal S, Garg S, Venkatesh P, Mithal C, Vohra R, Mehrota A: Intravitreal bevacizumab for subfoveal idiopathic choroidal neovascularization. Arch Ophthalmol 2007;125:1487-1492.

4 Chan WM, Lai TY, Liu DT, Lam DS: Intravitreal bevacizumab (avastin) for choroidal neovascularization secondary to central serous chorioretinopathy, secondary to punctuate inner chorioretinopathy, or of idiopathic origin. Am J Ophthalmol 2007;143:977-983.

-5 Vuorela P, Hatva E, Lymboussaki A, Kaipainen A, Joukov V, Persico MG, Alitalo K, Halmesmäki E: Expression of vascular endothelial growth factor and placenta growth factor in human placenta. Biol Reprod 1997;56:489-494.

6 Macular Photocoagulation Study Group: Argon laser photocoagulation for idiopathic neovascularization: results of a randomized trial. Arc Ophthalmol 1983;101:1358-1361.

7 Lindblom B, Andersson T: The prognosis of idiopathic choroidal neovascularization in persons younger than 50 years of age. Ophthalmology 1998;105:1816-1820. 